\title{
FCC-ee and the CR effects
}

\author{
Nelli Pukhaeva ${ }^{1, *}$ \\ ${ }^{1}$ Joint Institute for Nuclear Research, 141980 Dubna, Russia
}

\begin{abstract}
The proposed new FCC-ee $e^{+} e^{-}$colliders will be hosted in the Geneva area as the new $100 \mathrm{~km}$ tunnel will allow producing collisions at centreof-mass energies from 90 to $350 \mathrm{GeV}$ and beyond at several interaction points and will be used for a precision measurement at the $Z$ pole, $W W$ threshold, $H Z$ cross section maximum and $t \bar{t}$ threshold with an unequaled accuracy.

New measurements, which will be realized with large amount of data and at high energies accessible at the FCC-ee would allow a detailed study of the Color Reconnection phenomenon, especially in the hadronic $W W$ decays, and would be helpful for other studies, for example in search for these effects in $t \bar{t}$ and in Higgs decays.
\end{abstract}

\section{Introduction}

FCC (Future Circular Collider), which could be in a new $100 \mathrm{~km}$ circular tunnel in the Geneva area will host a lepton-lepton, a hadron-hadron, and a lepton-hadron colliders, respectively as the project evolves.

The first part will be the FCC-ee, which is formally called TLEP[1] (see Fig. 1) providing $e^{+} e^{-}$collisions at a high luminosity in order to enable precision studies of several fundamental elementary particles. It will produce collisions at centre-of-mass energies from 90 to $350 \mathrm{GeV}$ and beyond at several interaction points for making a precision measurement at the $Z$ pole, $W W$ threshold, $H Z$ cross section maximum and $t \bar{t}$ threshold with an unequaled accuracy[2].

The events will be as "clean" as at LEP, with no pile-up interactions and negligible beam background.

The new particle accelerators will be an instrument to understand the physics underlying new observations. It must be new phenomena, albeit at unknown energy scales, as shown by the evidence for non-baryonic dark matter, the cosmological baryon-antibaryon asymmetry and non-zero neutrino masses, which are all evidence for physics beyond the Standard Model.

The color reconnections (CR) issues will reappear at FCC-ee high-energy $e^{+} e^{-}$colliders. The hadronic and semileptonic $W W$ events will be studied to probe the impact of CR effects. Both the top and $W$ have decay lengths of order of $0.1 \mathrm{fm}$, and their decay products participate in the swapping of colors. The $t \bar{t}$ decays with hadronic final states will allow the study of the $\mathrm{CR}$ effects. The CR uncertainties thereby affect the precision of measurements of the Higgs boson properties, which is one of the primary purposes of future $e^{+} e^{-}$colliders.

\footnotetext{
*e-mail: nelli.pukhaeva@gmail.com
} 


\section{FCC-ee timescale and physics programme}

Today the various possibilities are on the table for the choice of the most appropriate machine(s) to analyze new phenomena ( $\mathrm{pp}, e^{+} e^{-}, \mu^{+} \mu^{-}$and $\gamma \gamma$ colliders and it seems that circular $e^{+} e^{-}$colliders have the best potential (Fig. 2).

The choice of the most appropriate machine(s) to analyze these new phenomena may be determined by historical precedents, which reveal the important role played by lower-energy precision measurements when establishing roadmaps for future discoveries with higherenergy machines.

In the late 1970's, precision measurements of neutral currents reinforced the prediction of the existence of the $W$ and $Z$ bosons and constrained the values of their masses. The $W$ and $Z$ bosons were then discovered in the early 1980's as was expected at the CERN $S p \bar{p} S$ collider with masses in the range predicted. Subsequently, the CERN LEP $e^{+} e^{-}$collider measured the properties of the $Z$ and $W$ bosons with a high precision in 1990's. These precise measurements led to the prediction of the top-quark mass, which was discovered at the FNAL Tevatron with the predicted mass. The measurement of $m_{t o p}$, together with the precise measurement of the $W$ mass at the Tevatron in the past decade, led in turn to a prediction of the mass of the Higgs boson, which was discovered at LHC within the predicted mass range.

The construction and installation of the collider and detectors of the FCC-ee and VHELHC setups could start and then proceed in parallel with the HL-LHC running for next five years with considerations to start commissioning for the first FCC physics run as early as 2030 .

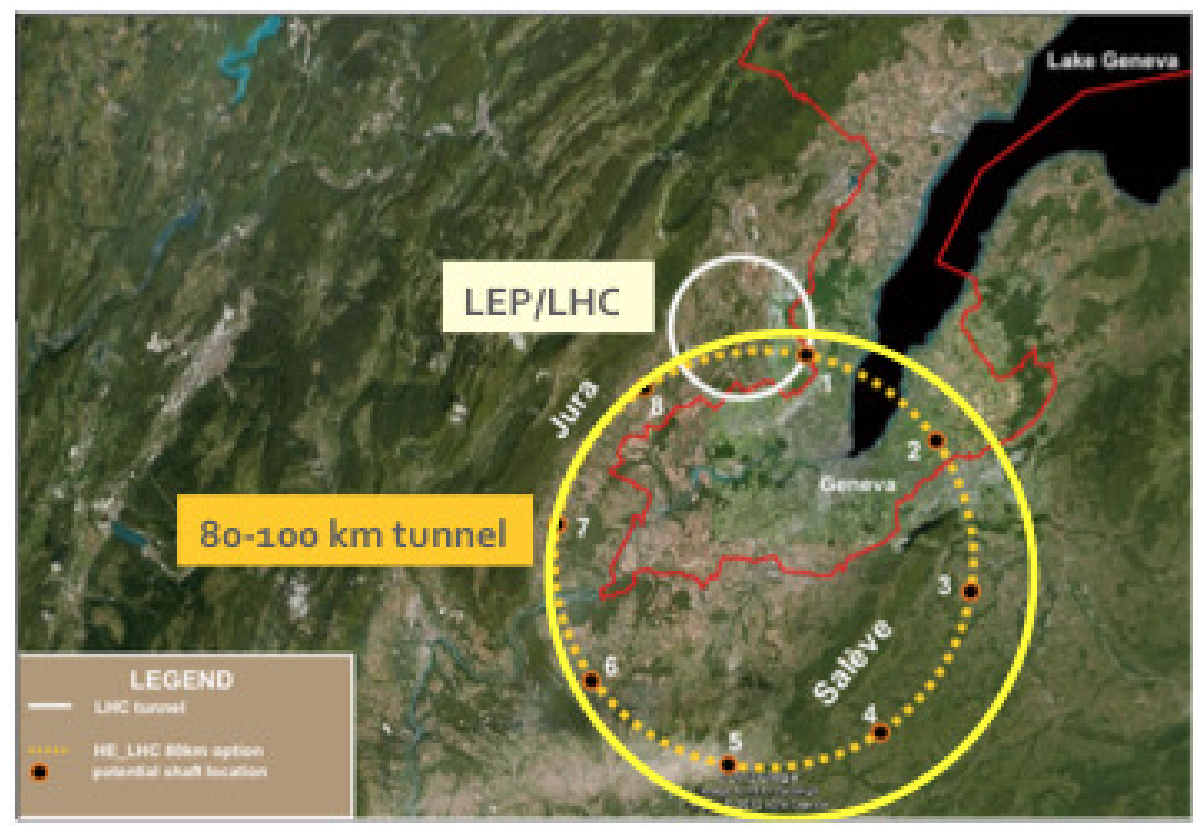

Figure 1. The $80 \mathrm{~km}$ tunnel (dashed circle) and the $100 \mathrm{~km}$ version (full line) that would host the TLEP and VHE-LHC in the Geneva area

The electroweak precision measurement made at LEP with $10^{7} Z$ decays, together with the accurate $W$ and top-quark mass measurement from the Tevatron, is sensitive to the new weakly-coupled physics at the scale up to $3 \mathrm{TeV}$. To increase this sensitivity up to $30 \mathrm{TeV}$, an 
improvement in precision by two orders of magnitude, i.e. an increase in statistics by four orders of magnitude to at least $10^{11} \mathrm{Z}$ decays, would be necessary [3].

The baseline of the physics programme of FCC-ee would consist of [4]

- two years at the $Z$ pole (one of which is the year with the designed luminosity of $5.6 a b^{-1}$ at each IP);

- one or two years at the $W W$ threshold with luminosity of $1.6 a b^{-1}$ per year at each IP and with periodic returns to the $Z$ peak;

- five years at $240 \mathrm{GeV}$ as a Higgs factory, with luminosity of $500 \mathrm{fb}^{-1}$ per year at each IP and with periodic returns to the $Z$ peak;

- five years at the $t \bar{t}$ threshold with the luminosity of $130 \mathrm{fb}^{-1}$ per year at each IP and with periodic returns to the $Z$ peak.

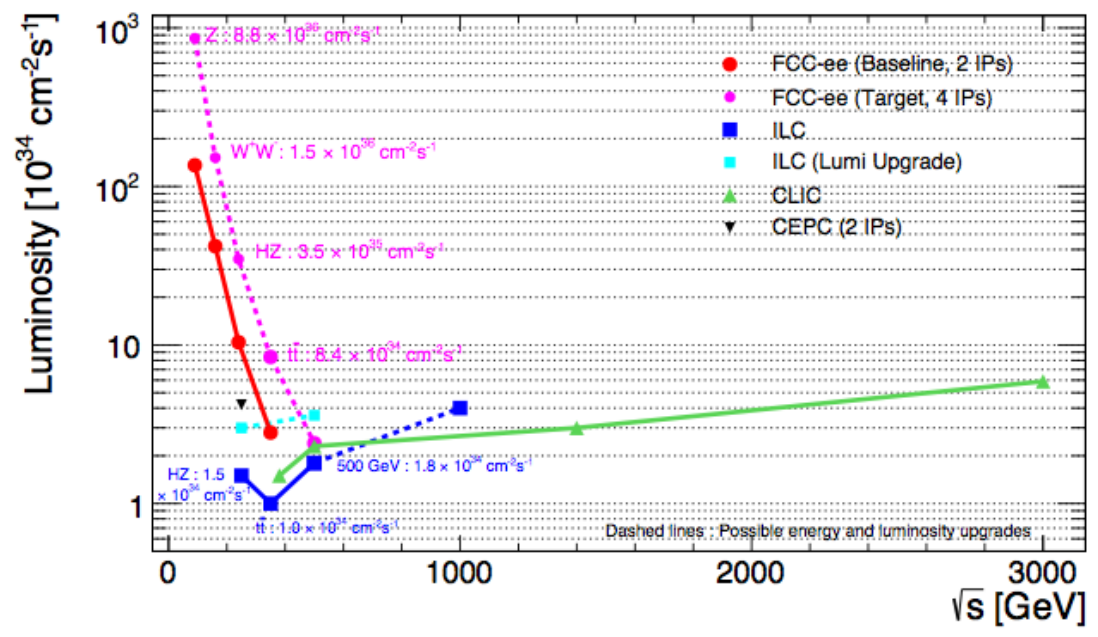

Figure 2. The luminosity expected at TLEP (full red line), with four interactions points, the luminosities expected at linear colliders, ILC (blue line) and CLIC (green line)

The Higgs boson coupling needs to be measured with a one-percent accuracy or better to be sensitive to the $1 \mathrm{TeV}$ new physics and with a permil accuracy to be sensitive to the multi-TeV new physics. In the Higgs decay channels to $W^{+} W^{-}$and $Z^{0} Z^{0}$, the fully hadronic decays would be sensitive to the CR between the two gauge-boson systems.

The FCC-ee will be a $W$ factory with more than $2 \times 10^{8} \mathrm{~W}$ pairs produced at center-ofmass energies at the $W W$ threshold and above. Since the quantity of data expected at the $W W$ production threshold is $10^{5}$ times larger than that produced at LEP, the measurements to be performed at the FCC-ee at these center-of-mass energies need to be thoroughly reviewed starting from the study of the design. The precision achieved at LEP on $m_{W}$ was about 300 $\mathrm{MeV}$ per experiment. A statistical error of $1 \mathrm{MeV}$ on the $W$ mass will be achievable at the FCC-ee per experiment and it would already be a very sensitive probe of new physics and would allow detailed tests of the CR phenomenon in the hadronic $W W$ channel.

The FCC-ee will be a top factory as well, with over one million $t \bar{t}$ pairs produced in five years at $345 \mathrm{GeV}$ at the $t \bar{t}$ threshold. 


\section{Nonperturbative QCD effects and hadronization}

Quantum Chromodynamics is the only unbroken Yang-Mills theory that can be compared directly with experiment. It exhibits the unique and complicating feature of self-interactions between massless gauge bosons (moreover with a tree-level coupling, which is relatively large) and, related to that, the still fundamentally puzzling feature of confinement. Its rich structure is probed in a particularly clean way by the $e e \rightarrow$ hadrons process, where quarks produced from the vacuum turn into (jets of) hadrons. At $\mathrm{CM}$ energies above a few $\mathrm{GeV}$, this process can be described via a combination of quark and gluon bremsstrahlung showers, which may be treated perturbatively and constrained by fits to data. These two components correspond to short and long wavelengths, respectively, and a hadronization process, which must be parameterized or modeled nonperturbatively and constrained by fits to data. The two components corresponding to short and long wavelengths may formally be factorized from each other. This is the basis of all calculations in QCD.

The confinement remains one of the fundamental unsolved problems in the quantum field theory. For infrared safe observables, its effects may be minimized and cast in terms of factorized and universal long-distance corrections (power corrections) and/or functions (FFs, PDFs). Monte-Carlo models (such as the string or cluster ones) attempt to go further and provide an explicit non-perturbative modeling of the hadronization process. The latter allows direct comparisons with data at the particle level. Moreover, comparing models based on qualitatively different assumptions with data would allow one (if done carefully) to extract knowledge about the properties of the nonperturbative dynamics, a famous example being the discovery of the 'string effect' in the early eighties[5]. In both cases, there are free parameters that must be extracted from fits (or 'tunes' in the Monte Carlo (MC) context) to data. In this context, the clean environment of $e^{+} e^{-}$collisions is a sine qua non.

\subsection{The CR effects models}

There are several new models proposed during the last years. New models were introduced to study uncertainties in the top mass measurement and study the effect of CR in top decays [6]. In the 'forces' model, the reconnection involving top decay products are performed as follows. Two sets of gluons are constructed, one containing the gluons produced in the top decays $g_{t}$ and the other containing the gluons from the rest of the reaction products $g_{r}$. Iterating over $g_{t}$ in random order, one forces the gluons from top to exchange colors with gluons from the rest of the event.

A second class of models is also introduced, GM (Gluon-Move) and swap models, which simulate reconnections between all gluons in the event. The swap model is a generalization of the 'smallest $\Delta \lambda$ ', where the $\Delta \lambda$ is a tunable parameter which expresses a CR strength.

The 'move' model works as follows. The final state gluon is attached to a string piece between partons, the string length $\Delta \lambda$ changes as a result of moving the gluon to another string piece between other partons.

The model SCI (Soft Color Interaction) [7], where CR is described as a stochastic color exchange between perturbative partons and a background color field, which modifies the color structure of hadronization, such that color singlet systems may appear well separated in rapidity.

It is the new QCD-based model, namely the CS model (Color-Space) [8], which allows strings to form not only between leading-color(LC)-connected partons, but also between specific non-LC-connected ones, following the combination rules that approximate the multijet structure of full-color QCD. The CS model was introduced to look for effects in the soft QCD, especially the baryon production. 
The new models implemented to study CR effects at hadronic colliders show different behaviors for $e^{+} e^{-}$. The CS model gives rise to very limited variations, while for the GM models even one specific scenario shows large enough deviations to be excluded by the LEP data. All these new models could be used for any process.

Modeling and testing of $\mathrm{CR}$ in hadronic collisions is rather more complicated. However, the estimation of the average transverse momentum with increasing charged multiplicity plays an important role.

The paper [9] discussed whether the QCD vacuum behaves like a superconductor and it was concluded that there are two possibilities. If it is like a type I superconductor, the string should correspond to a flux tube, similar to an elongated bag, with more or less homogeneous color electric field. The diameter of the flux tube should be of order of $1 \mathrm{fm}$ (a typical hadron bag diameter) and it is expected that the geometry of the flux tube would be determined only when the separation of the color charges reaches this order of magnitude. If the vacuum is like a type II superconductor, the string would correspond to a vortex line with a thin core which is surrounded by an exponentially falling electric field. The diameter of this field would be about $1 \mathrm{fm}$ but the size of the core could be much smaller. In this case geometry of the string would be determined already when the separation reaches the core size.

All those assumptions were introduced in the later version on PYTHIA8.

\section{The effects of CR at FCC-ee}

The measurements at LEP convincingly demonstrated that the color reconnections (e.g. between the two decay systems in hadronic $W W$ events) exist in nature, ruling out the no-CR scenario at $99.5 \%$ CL [10]. The effect became the topic of significant interest in particular since 'string drag' had a noticeable effect on extractions of the $W$ mass. However, apart from the conclusion that it exists and the exclusion of some of the most aggressive (and possibly unrealistic) scenarios, not much differential information is available to constrain new generations of models.

The issue has reappeared with potentially enhanced effects in hadronic collisions where it can affect the top mass extraction and significant effects may be indicated by soft-QCD distributions in both minimum-bias and underlying-event measurement.

High-statistics $e^{+} e^{-}$measurements are necessary to tell the other side of the story [13]. In particular, it was suggested [12] that with thousands of time more $W W$ events at the FCC-ee rather than at LEP, the $W$ mass issue could be turned around using the huge sample of semileptonic (in which no CR can occur between the two decay systems) processes to determine $M_{W}$ with a high precision and then one can use this extracted value as a constraint to measure $\mathrm{CR}$ in the fully hadronic ones. Other measurement include the particle flow between jets, or charges in the charged multiplicity as a function of topology. Complementary studies should, of course, also be performed in $e e \rightarrow t t$ and multi-jet states of hadronic $Z$ decays. The perspectives of pinning down the CR mechanism at the FCC-ee are, therefore, good.

\subsection{Interconnection effects at $W W$}

In order to investigate the color reconnection in hadronic $W W$ decays various studies were performed with LEP2 data in all four experiments. These studies are aimed to better understand the strong interaction mechanisms and to control systematic uncertainties which may arise from effects of the $W$ mass measurements (Fig. 3). Combining the results of the four LEP experiments with the full LEP2 statistics one can show that the most extreme CR models could be ruled out. However, not enough sdata were collected to definitely distinguish between the more moderate CR and no CR $[10,11]$ models. 


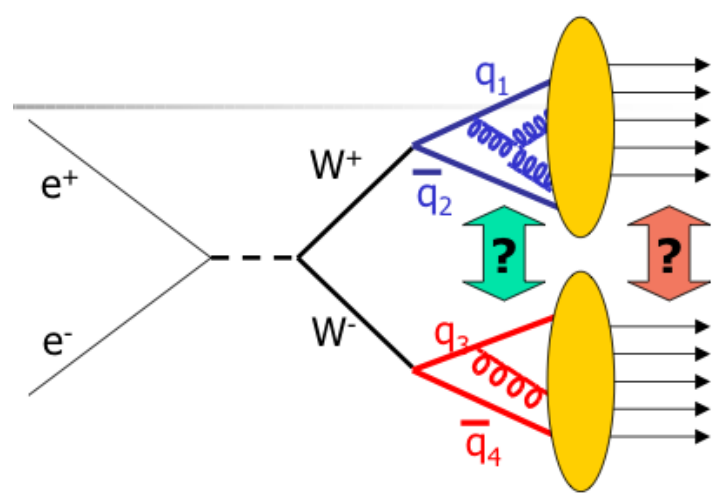

Figure 3. The effects of $\mathrm{CR}$ for $W W$ bosons in the string hadronization models

\subsection{Interconnection effects at $t \bar{t}$}

Since both the top and the $W$ bosons have decay lengths of order of $0.1 \mathrm{fm}$, their decay products participate in the swapping of colors. Similarly to the $W^{+} W^{-}$, the perturbative restructuring is suppressed because of the space-time separation between the decays of the $t \bar{t}$ quarks. At the perturbative level the rearrangement effects are negligibly small [14]. However, the non-perturbative aspects are not so well understood and there is no obvious reason why interconnection effects have to be small in the fragmentation process.

$t \bar{t}$ quark pairs are mainly produced through a QCD process. These pairs can be produced from either a pair of gluons or a pair of quarks in the initial state, Fig. 4. Top quarks are colored particles, the decay occurs before the hadronization and fragmentation owing to its large mass and width. Due to this effect, all its properties are transferred to its decays products, which are expected to be $99 \%$ of the cases a $W$ boson and a $b$ quark. Fully hadronic channels dominate and are about $44 \%$. Both $W$ bosons decay into a pair of quark and anti-quark of different flavor. As a result, six quarks are produced in total.

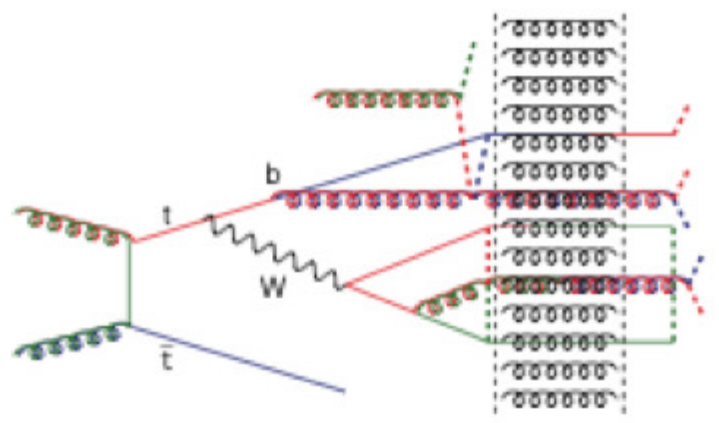

Figure 4. The top decay in the string hadronization models

There are up to six final-state quarks in $t \bar{t}$ events, in addition to the initial and final-state radiation. When hadronization and fragmentation occur, there are color interactions among these partons and color-remnants of the proton and antiproton. It changes the directions and distributions of final-state jets $[15,16]$ and affects the reconstructed value of $m_{t}[17,18]$. 
The effect of CR was studied at Tevatron for measured top masses [15, 19]. They used the Perudgia Tune Monte-Carlo model which included the Color Reconnection effects estimated from the LEP parameters obtained by the Professor collaboration [15] for underlying events. The uncertainty of the color reconnection was not evaluated for the Run I data because appropriate Monte-Carlo tools were not available that time. Both collaborations estimated this effect in the Run II by comparing the value of $m_{t}$ extracted from ensembles of $t \bar{t}$ events generated by PYTHIA using the difference between two parton shower simulations: A-PRO and ACR-PRO, where A-PRO is a model without color reconnection and ACR-PRO includes the color reconnection. The resulting uncertainties of $m_{t}$ are $0.32 \%$ for the CDF and $0.16 \%$ for the D0.

\subsection{CR in Higgs decays}

The Higgs state is very narrow (of order of $4 \mathrm{MeV}$ ) and very long-lived. The hadronization of the rest of the event already happened and the produced hadrons already spread out by the time when the Higgs boson decays. That mean that the Higgs boson itself decays essentially in a vacuum and has no interactions with the rest of the event neither in $e^{+} e^{-}$nor in $\mathrm{pp}$ collisions.

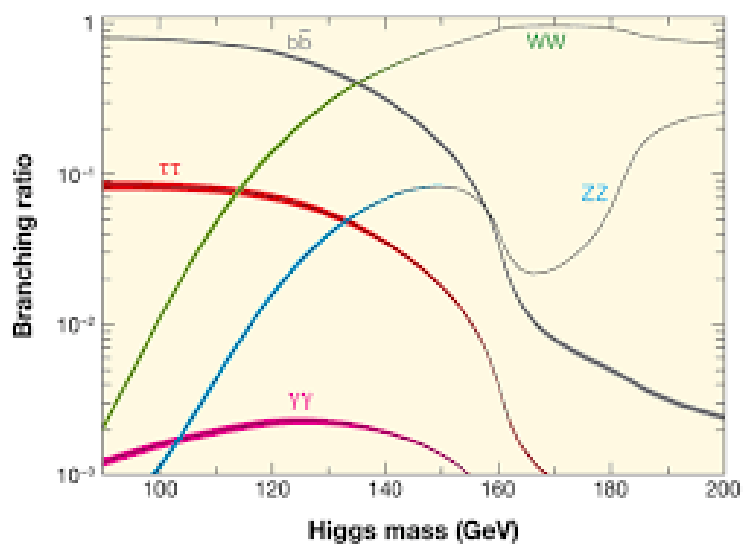

Figure 5. The Standard Model Higgs boson decay branching ratio

In the Standard Model, the Higgs boson is a pure $\mathrm{CP}$-even state, but in various extensions there can be a CP-odd admixture, and an important tack is to set stringent limits on this, Fig. 5. One possibility is to study angular correlations in $H^{0} \rightarrow W W \rightarrow q q q q$ decays [8]. The main point here is that $\mathrm{CR}$ also changes jet directions since the particle flow around a parton is biased in the direction towards its color partner due to standard string effects. This can give rise to deviations that could be misinterpreted, unless CR is well understood.

\section{Summary}

Studies following this approach should be continued. The study of the cross-talk phenomena for the $W$ mass reconstruction is important since it allows one to understand the scale of the corresponding ambiguities. The QCD reconnection is interesting as well since it may provide us with a prospective laboratory for probing the hadronization dynamics in space and time. 
Future $e^{+} e^{-}$colliders like FCC-ee will allow one to better understand and investigate these phenomena for the same classes of events, namely in the hadronic $W W$ channel. First results of the study of the $\mathrm{CR}$ effects at $e^{+} e^{-}$colliders were presented at the first workshop "Parton Radiation and Fragmentation from LNC to FCC-ee", which took place at CERN on November 21-22, 2016 [13]. The study of these phenomena at the FCC-ee energies will be continued using the new CR models which are implemented in the PYTHIA8 framework.

\section{References}

[1] M. Bicer et al. [TLEP design Working Group], JHEP 1401, 164 (2014)

[2] M. Koratzinos, A. Blondel, E. Gianfelice-Wendt and F. Zimmermann, arXiv:1506.00933.

[3] 1st FCC-ee workshop on precision observables and radiative corrections, July 2015, https://indico.cern.ch/event/387296/

[4] F. Zimmermann, 1st FCC annual workshop, Washington, March 2015, https://indico.cern.ch/event/340703/

[5] M. Green, J. Schwarz and E. Witten, Superstring Theory, (Cambridge University Press, 1987)

[6] S. Argyropoulos and T. Sjöstrand, JHEP 1411, 043 (2014)

[7] R. Enberg, G. Ingelman, N. Timneanu, Phys.Rev. D 64, (2001) 114015

[8] J. Christiansen, T. Sjöstrand, Eur.Phys.J. C 75(9), 441 (2015)

[9] G. Gustafson, U. Petterson and P. Zevas, Phys. Lett. B 209, 90 (1998)

[10] S. Schael et al., ALEPH, DELPHI, L3, OPAL, LEP Electroweak Collaboration, Phys.Rept. 532, 119-244 (2013)

[11] N.E. Pukhaeva, Phys.Part.Nucl.Lett. 15(5), $492-498$ (2018)

[12] D. d'Enterria, in Proc. of 18th LCEPPh, Moscow (2016) 182

[13] FCC-ee precision physics workshop, Feb. 2016, https://indico.cern.ch/event/469561/

[14] V.S. Fadin, V.A. Khose and A.D. Martin, Phys.Lett. B 320, 141 (1994)

[15] V.M. Abazov et al. (D0 Collaboration), Phys.Rev. D 83, (2011) 092002

[16] B.R. Webber, J.Phys.G: Nucl. Part. Phys. 24, 287 (1998)

[17] P.Skands and D. Wicke, Eur.Phys.J. C 52, 133 (2007)

[18] N.E. Pukhaeva, on Proceeding XIV APMWP, Grodno (2018)

[19] Aaltonen et al. (CDF and D0 Collaborations), Phys. Rev. D 86, 920 (2012) 